\title{
MODULES OVER SEMIHEREDITARY BEZOUT RINGS
}

\section{THOMAS S. SHORES}

\begin{abstract}
It is shown that every commutative semihereditary Bezout ring of Krull dimension at most one is an elementary divisor ring. A consequence is that the ring of polynomials in one indeterminate over a von Neumann regular ring is an elementary divisor ring.
\end{abstract}

All rings of this note are commutative with unity and modules are unital. Recently there has been some interest in the polynomial ring $R[X]$ where $R$ is a von Neumann regular ring. Such a ring is Bezout by Theorem 3.2 of [GP], semihereditary by [M], and so Hermite by Theorem 2.4 of [LLS]. Thus it is natural to ask whether or not $R[X]$ is an elementary divisor ring. This question is answered affirmatively by the Theorem below, which is a reduction technique for semihereditary Bezout rings. First we require the following Lemma, whose proof is contained in the proof of Theorem 3.8 of [LLS] and therefore omitted. Recall that a module is named by a matrix if it is isomorphic to the cokemel of the linear map between free modules defined by the matrix.

Lemma. The Hermite ring $R$ is an elementary divisor ring if and only if every module named by a matrix of the form $\left[\begin{array}{cc}p & 0 \\ r & q\end{array}\right]$, where $(p, q, r)=R$, is a direct sum of cyclic modules.

It has been pointed out to me by Roger Wiegand that if $R$ is a semihereditary Bezout ring, then a faithful module named by a matrix as in the Lemma can be shown to be projective by [B, p. 141], hence a direct sum of cyclics by a well-known theorem of Kaplansky on projective modules over semihereditary rings. However it is interesting to note that somewhat more is true and we take the occasion to record this fact.

Proposition. A finitely presented faithful two generator module $M$ over the semibereditary Bezout ring $R$ is a direct sum of cyclic modules.

Proof. By Theorem 2.4 of [LLS], $R$ is a Hermite ring. By hypothesis $M$

Received by the editors October 10, 1973.

AMS (MOS) subject classifications (1970). Primary 13C05; Secondary 13 F05.

Key words and phrases. Commutative von Neumann regular ring, polynomial ring, semihereditary ring, Hermite ring, Bezout ring, elementary divisor ring. 
can be named by a 2 by $n$ matrix $A$ with entries in $R$ for suitable $n$. Thus by Theorem 3.5 of $\left[K_{1}\right], A$ is equivalent to a matrix $\left[\begin{array}{ll}B & 0\end{array}\right]$, where $B=\left[\begin{array}{ll}p & 0 \\ r & q\end{array}\right]$ is 2 by $2 . M$ is al so named by $B$. One checks that $M p q=0$ directly (or notes that $p q=\operatorname{det} B$, the first Fitting invariant of $M)$. The faithfulness of $M \mathrm{im}$ plies that $p q=0$. Since $R$ is semihereditary, there is an idempotent $e \in R$ such that $p=e p$ and $q=(1-e) q$. It is easily seen that $M e$ is named by $e A$ as an $e R$-module and a similar remark applies to $M(1-e)$. The rings $e R$ and $(1-e) R$ are homomorphic images of $R$, so are Hermite. Hence there exist invertible matrices $P_{1} \in e R_{(2)}$ and $Q_{1} \in(1-e) R_{(2)}$ such that

$$
P_{1} e A=\left[\begin{array}{ll}
d_{1} & 0 \\
0 & 0
\end{array}\right] \text { and }(1-e) A Q_{1}=\left[\begin{array}{ll}
0 & 0 \\
0 & d_{2}
\end{array}\right] \text {. }
$$

Now set $P=(1-e) I+P_{1}$ and $Q=e I+Q_{1}$, where $I$ is the 2 by 2 identity matrix. Then $P$ and $Q$ are invertible el ements of $R_{(2)}$ such that

$$
P A Q=(1-e) A Q_{1}+P_{1} e A=\left[\begin{array}{ll}
d_{1} & 0 \\
0 & d_{2}
\end{array}\right] .
$$

It follows that $M \cong R / d_{1} R \oplus R / d_{2} R$.

Theorem. Let $R$ be a semihereditary Bezout ring. Then $R$ is an elementary divisor ring if and only if $R / d R$ is an elementary divisor ring for all nonzero divisors $d \in R$.

Proof. One way is obvious. So suppose $R$ is a semihereditary Bezout ring whose images by nonzero divisors are elementary divisor rings. By the Lemma we need only show that if $M$ is named by a 2 by 2 matrix then $M$ is a direct sum of cyclic modules. If $M$ is faithful we are done by the Proposition. Otherwise, note that finitely generated ideals of $R$ are projective, so finitely presented. So $R$ is coherent, whence $M$ is coherent by Theorem 2.1 of [C]. Hence cyclic submodules of $M$ are finitely presented, so have finitely generated annihilators in $R$. If $M$ is generated by $x$ and $y$, then $(0: M)=$ $(0: x) \cap(0: y)$. Thus coherence of $R$ and Theorem 2.2 of [C] imply that $(0: M)$ is finitely generated. So let $(0: M)=c R$. Let $e$ be an idempotent of $R$ such that $(0: c)=e R$. Then $M e$ is a faithful $e R$-module and $e R$ is semihereditary Bezout, so $M e$ is a direct sum of cyclics by the Proposition. On the other hand $M(1-e)$ is a finitely presented $(1-e) R$-module with annihilator $c R=$ $(1-e) c R$. The element $e+c=d$ is a nonzero divisor of $R$ and $R / d R$ is both isomorphic to $(1-e) R / c R$ and an elementary divisor ring. It follows that $M(1-e)$ is a direct sum of cyclic submodules and the proof is complete. 
Corollary. Every semihereditary Bezout ring $R$ of Krull dimension at most 1 is an elementary divisor ring.

Proof. A nonzero divisor $d$ of $R$ does not belong to any minimal prime of $R$, so $R / d R$ is a zero dimensional ring. Hence $R / \operatorname{rad}(d R)$ is a von Neumann regular ring. Such rings are known to be elementary divisor rings (e.g. [GH, p. 365]). Consequently $R / d R$ is an elementary divisor ring by Theorem 3 of $[H]$ and the result follows.

Examples. (1) (Kaplansky $\left[\mathrm{K}_{2}\right.$, p. 80]) Let $R$ be a one dimensional Bezout domain. Then $R$ is an elementary divisor ring.

(2) Let $R$ be von Neumann regular. Any prime ideal of $R[X]$ contracts to a maximal ideal $\mathbb{R}$ of $R$. Since $(R / \mathbb{R})[X]$ is a principal ideal domain, $R[X]$ has Krull dimension one and the Corollary applies.

(3) Any Hermite ring of finite Krull dimension for which the subspace $V(d)$ of $\operatorname{minspec}(R)$ is compact for all nonzero $d \in R$, is an el ementary divisor ring by a proof similar to that of the Corollary.

\section{REFERENCES}

[B] N. Bourbaki, Eléments de mathématique. Fasc. XXVII. Algèbre commutative. Chap. 1: Modules plats, Actualités Sci. Indust., no. 1290, Hermann, Paris, 1961. MR $36 \# 146$.

[C] S. U. Chase, Direct products of modules, Trans. Amer. Math. Soc. 97 (1960), 457-473. MR $22 \# 11017$.

[GH] L. Gillman and M. Henriksen, Some remarks about elementary divisor rings, Trans. Amer. Math. Soc. 82 (1956), 362-365. MR 18, 9.

[GP] R. Gilmer and T. Parker, Semigroup rings as Prüfer rings (preprint).

[H] M. Henriksen, Some remarks on elementary divisor rings. II, Michigan Math. J. $3(1955 / 56), 159-163$. MR 19, 1155.

[K, ] I. Kaplansky, Elementary divisors and modules, Trans. Amer. Math. Soc. 66 (1949), 464-491. MR 11, 155.

$\left[\mathrm{K}_{2}\right] \longrightarrow$, Infinite abelian groups, rev. ed., Univ. of Michigan Press, Ann Arbor, Mich., 1969. MR 38 \#2208.

[LLS] M. Larsen, W. Lewis and T. Shores, Elementary divisor rings and finitely presented modules, Trans. Amer. Math. Soc. 187 (1974), 231-248.

[M] P. J. McCarthy, The ring of polynomials over a von Neumann regular ring, Proc. Amer. Math. Soc. 39 (1973), 253-254.

DEPARTMENT OF MATHEMATICS, UNIVERSITY OF NEBRASKA, LINCOLN, NEBRASKA 68508 